\title{
Hydrogen and the Abundances of Elements in Gradual Solar Energetic-Particle Events
}

\author{
Donald V. Reames ${ }^{1}$
}

Received: 8 February 2019 / Accepted: 13 May 2019 / Published online: 5 June 2019

(C) The Author(s) 2019

\begin{abstract}
Despite its dominance, hydrogen has been largely ignored in studies of the abundance patterns of the chemical elements in gradual solar energetic particle (SEP) events; those neglected abundances show a surprising new pattern of behavior. Abundance enhancements of elements with $2 \leq Z \leq 56$, relative to coronal abundances, show a power-law dependence versus their average mass-to-charge ratio $A / Q$, which varies from event to event and with time during events. The ion charge states $Q$ depend upon the source plasma temperature $T$. For most gradual SEP events, shock waves have accelerated ambient coronal material with $T<2 \mathrm{MK}$ with decreasing power laws in $A / Q$. In this case, the proton abundances agree rather well with the power-law fits extrapolated from elements with $Z \geq 6$ at $A / Q>2$ down to hydrogen at $A / Q=1$. Thus the abundances of the elements with $Z \geq 6$ fairly accurately predict the observed abundance of $\mathrm{H}$, at a similar velocity, in most SEP events. However, for those gradual SEP events where ion enhancements follow positive powers of $A / Q$, especially those with $T>2 \mathrm{MK}$ where shock waves have reaccelerated residual suprathermal ions from previous impulsive SEP events, proton abundances commonly exceed the extrapolated expectation, usually by a factor of order ten. This is a new and unexpected pattern of behavior that is unique to the abundances of protons and may be related to the need for more streaming protons to produce sufficient waves for scattering and acceleration of more heavy ions at the shock.
\end{abstract}

Keywords Solar energetic particles - Solar system abundances - Coronal mass ejections · Solar flares

\section{Introduction}

Hydrogen is, by far, the most abundant element in typical astrophysical settings, including the Sun, where the pattern of element abundances is believed to have changed little during

D.V. Reames

dvreames@umd.edu

1 Institute for Physical Science and Technology, University of Maryland, College Park, MD 20742-2431, USA 
the 4.6 billion years since its formation. As elements evaporate from the solar photosphere up into the corona, those that are ionized, i.e. with first ionization potential (FIP) $<10 \mathrm{eV}$, are preferentially enhanced by a factor of about four relative to the elements with FIP $>10 \mathrm{eV}$, which are initially neutral atoms. Once in the 1-MK corona, all elements are highly ionized, and at altitudes where the plasma becomes collisionless, ion acceleration by electromagnetic fields can be sustained. The effects of those fields on acceleration and transport depends upon the magnetic rigidity of the ions, which, when compared at a constant velocity, varies as the ion mass-to-charge ratio $A / Q$. Thus it is not surprising that the effects of acceleration and transport on the abundances of solar energetic particles (SEPs) are found to vary as power laws in $A / Q$. The surprise is that scientists have advanced this picture of abundance variations of the elements for decades without including the abundance of hydrogen. Do protons ever fit this abundance pattern?

Early measurement of abundances, averaged over the large SEP events we now call "gradual" events, began to show clear evidence of the FIP effect (e.g. Webber, 1975; Meyer, 1985; Reames, 1995, 2014) especially for the elements from He or C through Fe. Once typical element ionization states $Q$ first became available (Luhn et al., 1984), event-to-event variations were found to be power-law functions of $A / Q$ for elements with atomic numbers $6 \leq Z \leq 30$ by Breneman and Stone (1985), but $\mathrm{H}$ was also omitted from more recent similar studies by Reames (2016a, 2016b, 2018b). Even the inclusion of He is rather complicated (Reames, 2017c). Power-law behavior of abundances in "impulsive" SEP events was proposed by Reames, Meyer, and von Rosenvinge (1994) using abundances of elements with $2 \leq Z \leq 26$ and the extension to high $Z$ shows an especially strong behavior as the third power of $A / Q$ for $2 \leq Z \leq 82$ (Reames, 2000, 2017a; Mason et al., 2004; Reames and Ng, 2004; Reames, Cliver, and Kahler, 2014a).

The terms "impulsive" and "gradual" have come to refer to the dominant physical process of particle acceleration in these SEP events as evidence for two mechanisms has evolved (Reames, 1988, 1999, 2013, 2015, 2017a; Gosling, 1993). The evidence of unique physics in the small impulsive SEP events was first shown by huge 1000fold enhancements of ${ }^{3} \mathrm{He} /{ }^{4} \mathrm{He}$ (Serlemitsos and Balasubrahmanyan, 1975; Mason, 2007; Reames, 2017a). While ${ }^{3} \mathrm{He} /{ }^{4} \mathrm{He} \approx 5 \times 10^{-4}$ is found in the solar wind, even the early measurements found SEP events with ${ }^{3} \mathrm{He} /{ }^{4} \mathrm{He} \approx 1.5 \pm 0.1$. A few events even have ${ }^{3} \mathrm{He} / \mathrm{H}>1$ (Reames, von Rosenvinge, and Lin, 1985). The events were associated with streaming nonrelativistic electrons and type III radio bursts (Reames, von Rosenvinge, and Lin, 1985; Reames and Stone, 1986). In the most complete theory, the streaming electrons produce electromagnetic ion-cyclotron (EMIC) waves in resonance with the gyrofrequency of ${ }^{3} \mathrm{He}$ to produce the unique enhancements (Temerin and Roth, 1992; Roth and Temerin, 1997). Further complicating the behavior, power-law enhancements of elements, also 1000-fold, between $\mathrm{He}$ and the heavy elements up to $\mathrm{Pb}$ (Reames, 2000, 2017a; Mason et al., 2004; Reames and Ng, 2004; Reames, Cliver, and Kahler, 2014a) were found and were better explained by particle-in-cell simulations of magnetic reconnection (Drake et al., 2009), which produce the power-law dependence on $A / Q$ from rigidity dependence during acceleration. Impulsive SEP events are associated with narrow coronal mass ejections (CMEs) related to solar jets (Kahler, Reames, and Sheeley, 2001; Bučík et al., 2018) driven by magnetic reconnection involving open field lines.

In gradual or long-duration SEP events, particles are accelerated at shock waves driven out from the Sun by fast, wide CMEs (Kahler et al., 1984). These shock waves accelerate SEPs (Lee, 1983, 2005; Zank, Rice, and Wu, 2000; Cliver, Kahler, and Reames, 2004; $\mathrm{Ng}$ and Reames, 2008; Gopalswamy et al., 2012; Lee, Mewaldt, and Giacalone, 2012; Desai and Giacalone, 2016; Reames, 2017a) requiring shock speeds above $500-700 \mathrm{~km} \mathrm{~s}^{-1}$ 
(Reames, Kahler, and Ng, 1997), and accelerating ions over an extremely broad region of the heliosphere (Reames, Barbier, and Ng, 1996; Rouillard et al., 2012; Cohen, Mason, and Mewaldt, 2017; Reames, 2017b). Generally, the shock waves sample the 1-2 MK coronal plasma (Reames, 2016a, 2016b, 2018b). Power-law dependence in gradual events can come from rigidity-dependent scattering of ions during transport (Parker, 1963; Ng, Reames, and Tylka, 1999, 2001, 2003, 2012; Reames, 2016a, 2016b), which results in dependence upon $A / Q$ when ion abundances are compared at the same particle velocity. If Fe scatters less than $\mathrm{O}$ in transit, for example, $\mathrm{Fe} / \mathrm{O}$ will increase early in an event and be depleted later on; this dependence on time and space traveling along the field $\mathbf{B}$ also becomes longitude dependent because of solar rotation.

However, SEP abundances become complicated because shock waves can also reaccelerate residual ions from a seed population that includes pre-accelerated ions from the ubiquitous small impulsive SEP events, complicating the SEP abundance story. Mason, Mazur, and Dwyer (2002) noted the presence of modest enhancements of ${ }^{3} \mathrm{He}$ in large SEP events that would otherwise be called gradual and the complexity of the seed population was soon realized (Desai et al., 2003; Tylka et al., 2005; Tylka and Lee, 2006; Bučík et al., 2014, 2015, 2018; Chen et al., 2015; Reames, 2016a, 2016b). Thus gradual SEP events sometimes exhibit the abundance enhancement patterns of impulsive SEP events, which we can recognize from power-law enhancements increasing with $A / Q$ and source plasma temperatures of $\approx 3$ MK (Reames, Cliver, and Kahler, 2014a, 2014b, 2015; Reames, 2019a, 2019b), which actually dominate $24 \%$ of gradual events (Reames, 2016a, 2016b). The ${ }^{3} \mathrm{He} /{ }^{4} \mathrm{He}$ ratio is also enhanced in these events (Reames, Cliver, and Kahler, 2014b) but its extreme variation with energy and from event to event (Mason, 2007) makes Fe/O a more stable and reliable indicator of impulsive material. Hence, we prefer $\mathrm{Fe} / \mathrm{O}$ rather than ${ }^{3} \mathrm{He} /{ }^{4} \mathrm{He}$ to identify impulsive SEP events and reaccelerated impulsive ions (Reames, Cliver, and Kahler, 2014a). Recently, the abundance patterns of $\mathrm{H}$ (Reames, 2019b) and ${ }^{4} \mathrm{He}$ (Reames, 2019a) have been found for impulsive SEP events.

Protons have been studied in many aspects of gradual SEP events (e.g. Reames, 2017a), including acceleration (Lee, 1983, 2005; Zank, Rice, and Wu, 2000; Ng and Reames, 2008), transport (Parker, 1963; Ng, Reames, and Tylka, 1999, 2001, 2003, 2012), time variations (Reames, Ng, and Tylka, 2000; Reames, 2009), and energy spectra (Reames and $\mathrm{Ng}, 2010$ ), but not for FIP or power-law abundance patterns. If we seek guidance from the case of the solar wind, we find variations are seen in $\mathrm{H} / \mathrm{He}$ with solar-wind speed and phase in the solar cycle (Kasper et al., 2007). However, while the solar wind is coronal in origin and does display a FIP effect (e.g. Schmelz et al., 2012), it is clear that SEPs have a different source than the solar wind (Mewaldt et al., 2002; Desai et al., 2003; Reames, 2018a), which may be related to differing open- and closed-field origins (Laming, 2009, 2015) of the two coronal samples (Reames, 2018a, 2018b). For our purposes, the SEP reference coronal abundances (derived from averaged gradual SEP events) and the solar photospheric abundances are provided in Appendix. We define an element "enhancement" to be its observed abundance, normalized to $\mathrm{O}$, divided by its corresponding reference abundance, similarly normalized.

A recent study of impulsive SEP events extended the power-law dependence of abundances on $A / Q$ from elements with $Z>6$ down to protons at $A / Q=1$ (Reames, 2019b). It was found that observed proton enhancements generally agreed well with the extrapolated value for small events with no evidence of shock acceleration. However, for most of the larger events, especially those associated with fast CMEs, observed proton intensities were a factor of order ten larger than expected. These impulsive SEP events are associated with solar jets where narrow CMEs can be fast enough to drive shock waves. It seems that 
the proton excess can be a signature of the presence of shock acceleration in impulsive SEP events. In all impulsive SEP events, abundance enhancements increase as positive powers of $A / Q$.

To what extent can we organize the abundance of $\mathrm{H}$ within the scheme of the other elements in gradual SEP events? We consider the gradual events studied and listed by Reames (2016a) using observations made by the Low-Energy Matrix Telescope (LEMT) on the Wind spacecraft, near Earth (von Rosenvinge et al., 1995; Reames et al., 1997; see also Chapter 7 of Reames, 2017a). LEMT primarily measures elements from He through $\mathrm{Pb}$ in the region of 2-20 MeV amu${ }^{-1}$. The element resolution of LEMT up through $\mathrm{Fe}$ is shown in detail by Reames (2014). LEMT resolves element groups above Fe as shown by Reames (2000, 2017a). Unlike other elements, the protons in LEMT are limited to a small interval sampled near $\approx 2.5 \mathrm{MeV}$ bounded by the front-detector (dome) threshold (von Rosenvinge et al., 1995; Reames et al., 1997).

Throughout this text, whenever we refer to the element He or its abundance, we mean ${ }^{4} \mathrm{He}$, unless ${ }^{3} \mathrm{He}$ is explicitly stated.

\section{Power-Laws in $A / Q$}

The theory of diffusive transport provides support for our expectation that element enhancements in gradual events will vary approximately as power laws in $A / Q$. It is common to expect that the scattering mean free path $\lambda_{\mathrm{X}}$ of species $\mathrm{X}$ depends as a power law on the particle magnetic rigidity $P$ as $P^{\alpha}$ and upon distance from the $\operatorname{Sun} R$ as $R^{\beta}$ so that the expression for the solution to the diffusion equation (Equation C3 in Ng, Reames, and Tylka, 2003, based on Parker, 1963) can be used to write the enhancement of element X relative to oxygen as a function of time $t$ as

$$
\frac{\mathrm{X}}{\mathrm{O}}=L^{-\frac{3}{(2-\beta)}} \exp \left\{\left(1-\frac{1}{L}\right) \frac{\tau}{t}\right\} r^{S},
$$

where $L=\lambda_{\mathrm{X}} / \lambda_{\mathrm{O}}=r^{\alpha}=\left(\left(A_{x} / Q_{x}\right) /\left(A_{\mathrm{O}} / Q_{\mathrm{O}}\right)\right)^{\alpha}$ and $\tau=3 R^{2} /\left[\lambda_{\mathrm{O}}(2-\beta)^{2} v\right]$ for particles of speed $v$. Since we compare different ions at the same velocity, their rigidities can be replaced by their corresponding values of $A / Q$. The factor $r^{S}$ is included to represent any $A / Q$-dependent power-law enhancement at the source. For shock acceleration of impulsive suprathermal ions, it describes the power-law enhancement of the seed particles expected from acceleration in impulsive SEP events (e.g. Drake et al., 2009). For shock acceleration of the ambient coronal material, $S=0$.

To simplify Equation 1, we can achieve a power-law approximation if we expand $\log x=$ $(1-1 / x)+(1-1 / x)^{2} / 2+\cdots$ (for $\left.x>1 / 2\right)$. Using only the first term to replace $1-1 / L$ with $\log L$ in Equation 1, we have

$$
\frac{\mathrm{X}}{\mathrm{O}} \approx L^{\frac{\tau}{t}-\frac{3}{(2-\beta)}} r^{S}
$$

as an expression for the power-law dependence of enhancements on $A / Q$ for species $\mathrm{X}$.

More generally, we can write Equation 2 in the form $\mathrm{X} / \mathrm{O}=r^{p}$, where the exponent $p$ is linear in the variable $1 / t$, so that

$$
p=\alpha \tau / t+S-3 \alpha /(2-\beta)
$$


Figure 1 Observed element enhancements (left) are compared with theoretical plots of $A / Q$ versus $T$ (right) for 8 -hr periods in 14 November 1998 (upper panels) and 22 May 2013 (lower panels) SEP events.

Temperatures are chosen that best match the observed groupings of elements (Reames, 2016a).
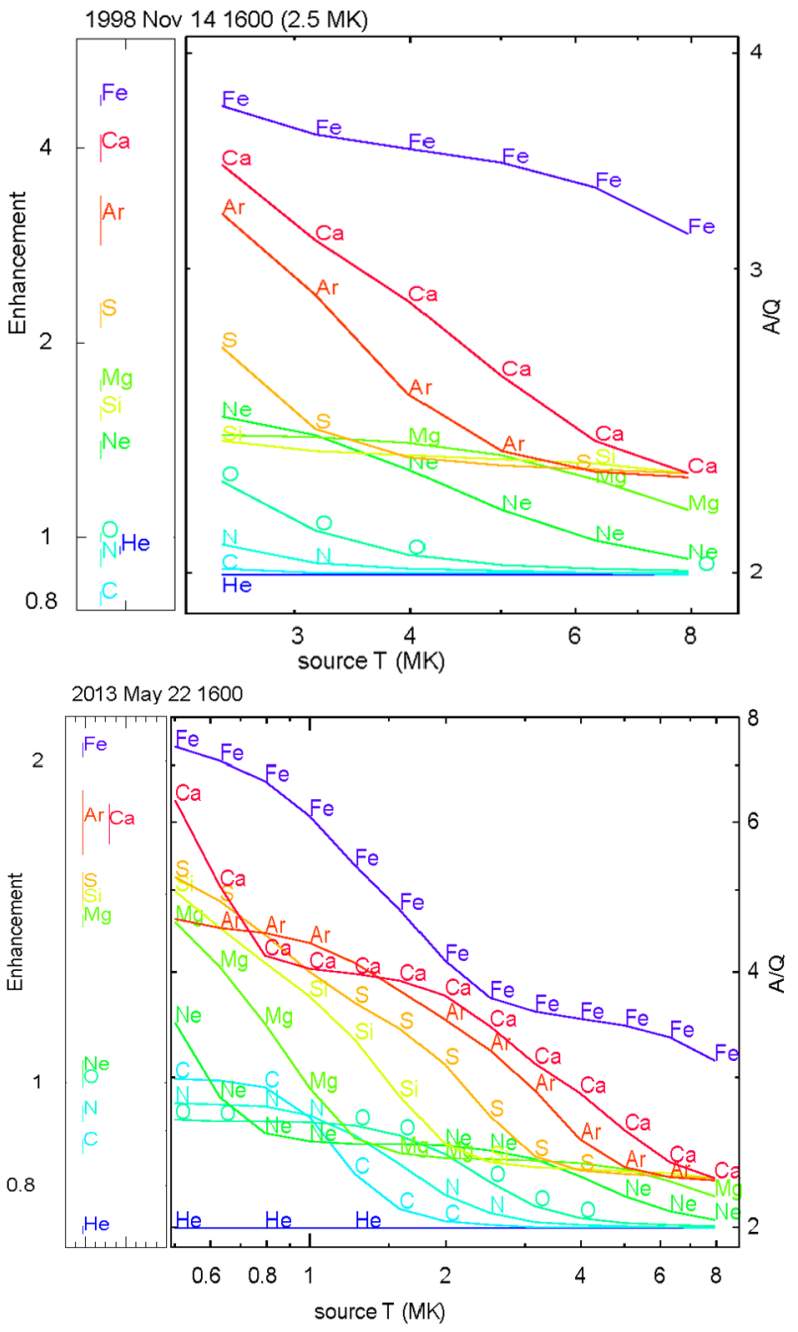

the average parameters are directly measurable from the time behavior of SEP-abundance observations (Reames, 2016b). Fits to the time behavior of typical gradual SEP events show that late in events where impulsive material is not present $p \approx-1$ to -2 (Reames, 2016b), while the average power law for impulsive events suggests $S \approx 3$ (Reames, Cliver, and Kahler, 2014a; Mason et al., 2004). The ions in small impulsive events generally propagate scatter-free (Mason et al., 1989), but in the more intense gradual events any shockaccelerated impulsive suprathermal ions are scattered, so that the power for the heavyelement enhancements is reduced (Reames, 2016b).

Thus we can expect that the enhancements will vary as a power law in $A / Q$, but the pattern of the average value of $Q$ for each element depends upon the source plasma temperature $T$ of the ions. Figure 1 shows two examples of observed abundance enhancements for SEP events matched against regions of the theoretical plots of $A / Q$ versus $T$ based upon Mazzotta et al. (1998). 
For the event at $T \approx 2.5 \mathrm{MK}$ in the upper left panel of Figure 1, the almost fully ionized elements $\mathrm{C}, \mathrm{N}$, and $\mathrm{O}$ occur grouped with $\mathrm{He}$, while $\mathrm{Ne}, \mathrm{Mg}$, and $\mathrm{Si}$, with two orbital electrons, are grouped at an interval above them. For the example in the lower panels, with $T \approx 0.7 \mathrm{MK}$, partially ionized $\mathrm{C}, \mathrm{N}$, and $\mathrm{O}$ have moved well above $\mathrm{He}$ and are now grouped with $\mathrm{Ne}$, while $\mathrm{Mg}$ and $\mathrm{Si}$ have moved far above $\mathrm{Ne}$ and have joined $\mathrm{S}$ approaching $\mathrm{Ar}$ and $\mathrm{Ca}$. We will see that the $\chi^{2}$ values of fits can be quite sensitive to these variations.

Given a temperature and the associated values of $A / Q$, we can determine, by leastsquares fitting, the best fit line and the associated value of $\chi^{2} / m$, where $m$ is the number of degrees of freedom. Repeating this fitting for every $T$ of interest, we can plot $\chi^{2} / m$ versus $T$ (e.g. Figure 2d) to choose the most likely temperature and fit line (Reames, 2016a, 2016b, 2018 b). In the present article, we include only the elements $Z \geq 6$ in the fits, and compare the best fit line with $\mathrm{He}$ and extrapolate it to $\mathrm{H}$. In the fits an error of $15 \%$ is assumed for random abundance variations in addition to the statistical errors.

\section{Low- $T$ Events and Decreasing Powers of $A / Q$}

Guided by the power-law fits of gradual events for $Z \geq 2$ by Reames (2016a), we first examine events with $T<2 \mathrm{MK}$, which seem to represent shock acceleration of the ambient solar coronal plasma.

Figure 2 shows the analysis of the event of 24 August 1998 from a source at longitude E09 on the Sun. The extrapolated values of the least-squares power-law fits for elements with $Z \geq 6$ for the six 8-hr time intervals agree rather well with the measured enhancement for $\mathrm{H}$ plotted at $A / Q=1$. In this event, the abundances of the elements with $Z \geq 6$ actually predict the abundances of $\mathrm{H}$ and $\mathrm{He}$.

Figure 3 shows the event of 23 January 2012 from a source at W21 on the Sun with an associated CME speed of $2175 \mathrm{~km} \mathrm{~s}^{-1}$. The enhancements versus $A / Q$ are quite flat during the early periods so $T$ is poorly determined. Here again the abundance of $\mathrm{H}$ is fairly well predicted by the abundances of elements with $Z \geq 6$.

Figure 4 shows analysis of the first two of the Halloween events on 26 and 28 October 2003 from W38 and E08 with CME speeds of 1537 and $2459 \mathrm{~km} \mathrm{~s}^{-1}$, respectively. Extrapolation of the least-squares fits for $Z \geq 6$ to $A / Q=1$ fit $\mathrm{H}$ rather well, even when the slope reverses for the last interval in Figure 4e. These events have been studied previously for their protons (Mewaldt et al., 2005) and their heavier ions (Cohen et al., 2005) separately.

\section{High- $T$ Events with Increasing Powers of $A / Q$}

$\mathrm{H}$ seemed to fit the abundance pattern of the preceding events. However, there is another class of events where it does not; examples have already been seen for impulsive SEP events (Reames, 2019b). Events with source plasma temperatures in the 2.5-3.2 MK range and positive power-law increase with $A / Q$ have been associated with shock reacceleration of impulsive suprathermal ions (Reames, 2016a, 2016b, 2017c, 2018b). In our sample of 45 gradual SEP events with measurable source plasma temperatures, $11(24 \%)$ were of this class (Reames, 2016a). These events have very small variations in abundance ratios, especially $\mathrm{C} / \mathrm{He}$ and $\mathrm{O} / \mathrm{C}$, compared with impulsive SEP events (Reames, 2016b). Since He, C, and $\mathrm{O}$ are nearly fully ionized at these temperatures, the small variations suggest that these "hot" gradual events have averaged abundances over a seed population consisting of many small impulsive SEP contributions (Reames, 2016b). 


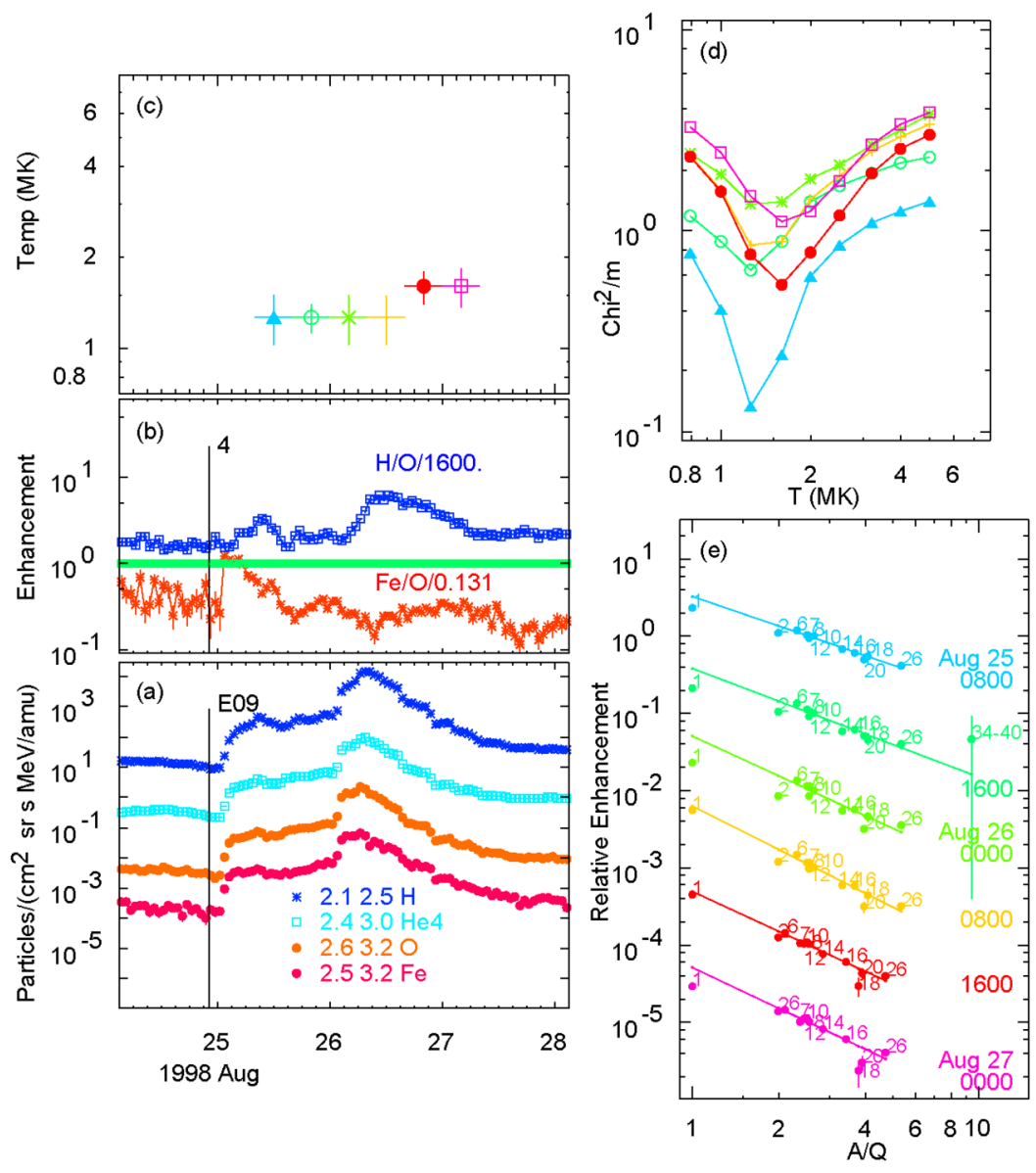

Figure 2 (a) Intensities of $\mathrm{H}, \mathrm{He}, \mathrm{O}$, and Fe versus time. (b) Normalized abundance enhancements $\mathrm{H} / \mathrm{O}$ and $\mathrm{Fe} / \mathrm{O}$ versus time. (c) Best-fit temperatures are shown versus time for the 24 August 1998 SEP event. (d) Shows $\chi^{2} / m$ versus $T$ for each 8 -hr interval. (e) Shows enhancements, labeled by $Z$, versus $A / Q$ for each 8 -hr interval shifted $\times 0.1$, with best-fit power law for elements with $Z \geq 6$ extrapolated down to $\mathrm{H}$ at $A / Q=1$. Colors correspond to the six intervals in (c), (d), and (e) and symbols in (c) and (d); times are also listed in (e). Event onset is flagged with solar longitude in (a) and event number from Reames (2016a) in (b).

Figure 5 is an example of an event with positive power-law slope and $T \approx 2.5 \mathrm{MK}$ from W63 on the Sun with a CME speed of $1556 \mathrm{~km} \mathrm{~s}^{-1}$. These properties suggest the presence of impulsive suprathermal ions with $S>0$ in the seed population. The proton enhancement in this event is an order of magnitude higher than expected from the extrapolation of the fit for the ions with $Z \geq 6$. This behavior was seen recently for the impulsive events where there is additional reacceleration of ions by shock waves (Reames, 2019b) and we will find this behavior common for many gradual SEP events with positive powers of $A / Q$ and with $T>2 \mathrm{MK}$, since they are dominated by reaccelerated impulsive SEP ions.

Figure 6 shows another example of an event with a positive power-law slope and $T \approx 3 \mathrm{MK}$, that of 14 November 1998 from a source behind the limb at W120. 


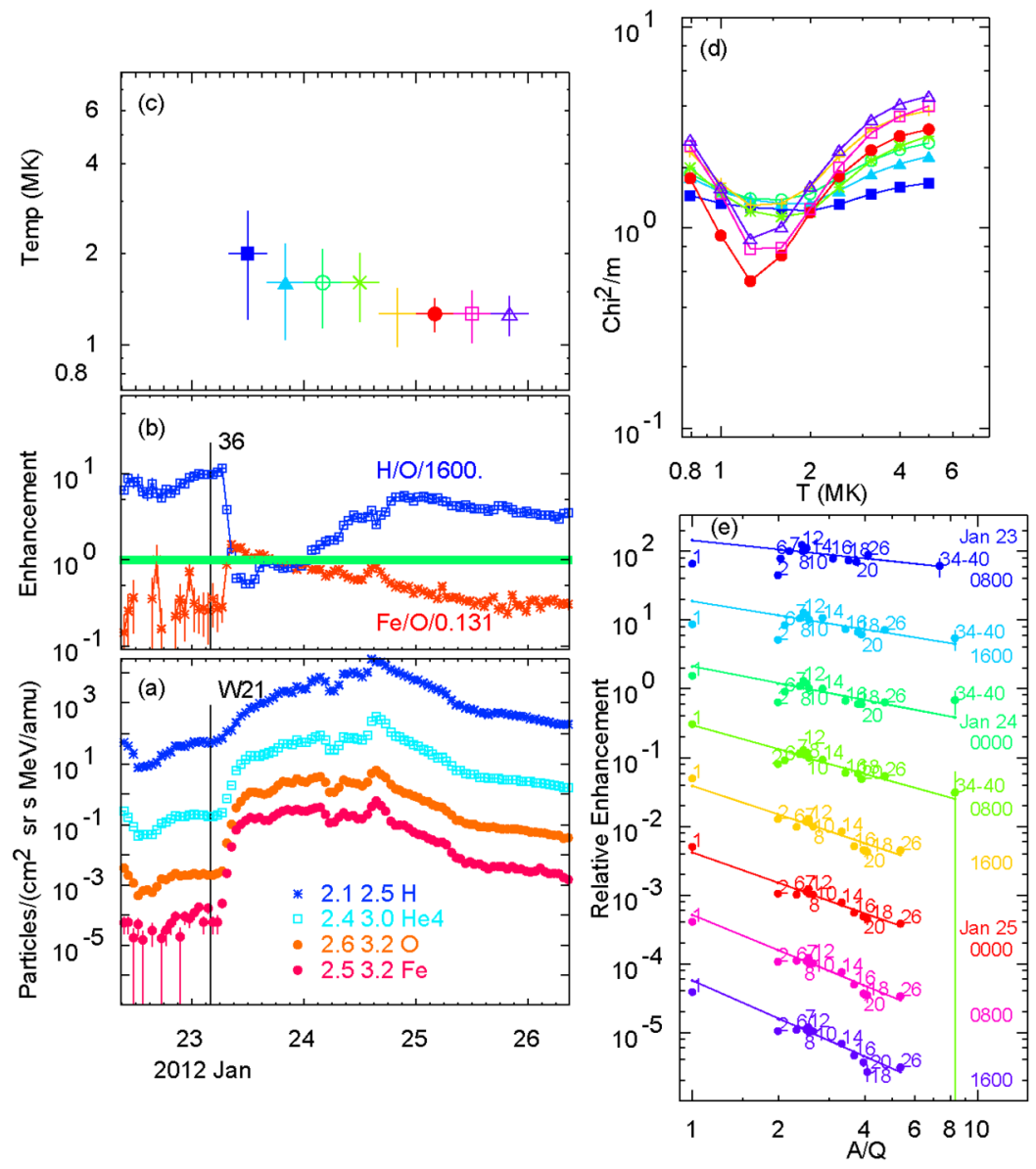

Figure 3 (a) Intensities of $\mathrm{H}, \mathrm{He}, \mathrm{O}$, and Fe versus time. (b) Normalized abundance enhancements $\mathrm{H} / \mathrm{O}$ and $\mathrm{Fe} / \mathrm{O}$ versus time. (c) Best-fit temperatures versus time for the 23 January 2012 SEP event. (d) Shows $\chi^{2} / m$ versus $T$ for each 8-hr interval. (e) Shows enhancements, labeled by $Z$, versus $A / Q$ for each 8-hr interval shifted $\times 0.1$, with best-fit power law for elements with $Z \geq 6$ extrapolated down to $\mathrm{H}$ at $A / Q=1$. Colors correspond to the eight intervals in (c), (d), and (e) and symbols in (c) and (d); times are also listed in (e). Event onset is flagged with solar longitude in (a) and event number from Reames (2016a) in (b).

For comparison, we show in Figure 7 the next event in the series of Halloween events, immediately following the events in Figure 4. The source of this event is a CME with a speed of $2029 \mathrm{~km} \mathrm{~s}^{-1}$ from longitude W02 on the Sun on 29 October 2003.

Unlike its immediate predecessors (shown in Figure 4) the event in Figure 7 shows a 2.5 MK seed population of impulsive SEP ions with an order-of-magnitude excess of protons. This event is not influenced by the properties of its immediate predecessor in Figure 4.

\section{Other Variations}

From the foregoing examples, one might conclude that hydrogen enhancements agree with those of other elements when the shock waves accelerate ambient coronal material and $\mathrm{H}$ ex- 


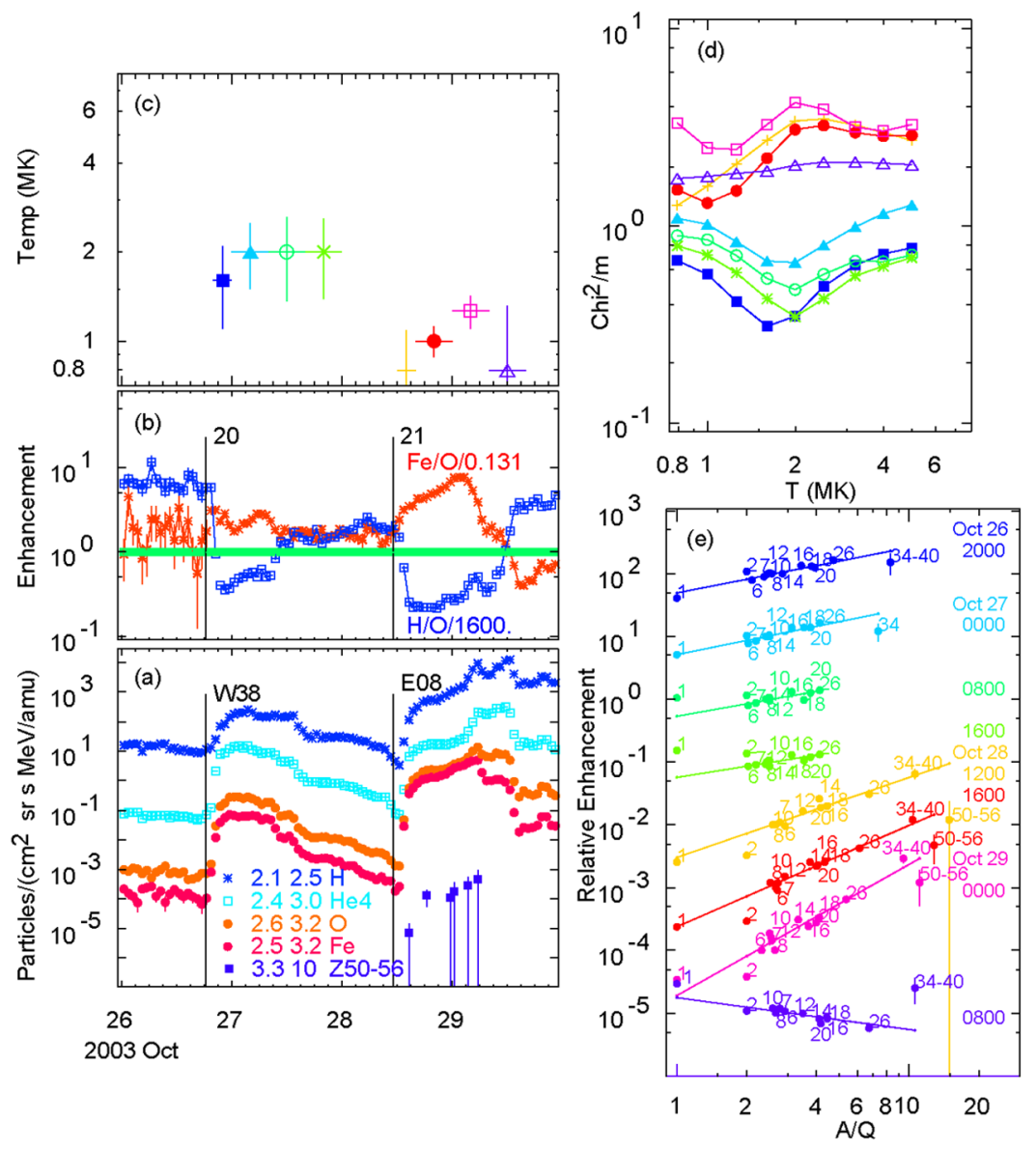

Figure 4 (a) Intensities of $\mathrm{H}, \mathrm{He}, \mathrm{O}, \mathrm{Fe}$, and $50 \leq Z \leq 56$ ions versus time. (b) Normalized abundance enhancements $\mathrm{H} / \mathrm{O}$ and $\mathrm{Fe} / \mathrm{O}$ versus time. (c) Derived source temperatures versus time, all for the 26 and 28 October 2003 SEP events. (d) Shows $\chi^{2} / m$ versus $T$ for each 8 -hr interval. (e) Shows enhancements, labeled by $Z$ versus $A / Q$ for each 8 -hr interval shifted $\times 0.1$ with best-fit power law for elements with $Z \geq 6$ extrapolated down to $\mathrm{H}$ at $A / Q=1$. Colors correspond to the eight intervals in (c), (d), and (e) and symbols in (c) and (d); times are also listed in (e). Event onsets are flagged with solar longitude in (a) and event number from Reames (2016a) in (b).

ceeds expectations by a factor of about 10 when impulsive SEP ions are reaccelerated. That does describe most gradual SEP events. However, there are some events with combinations of behavior that are more complex.

Figure 8 shows the analysis of the event of 2 April 2001 driven by a CME with a speed of $2505 \mathrm{~km} \mathrm{~s}^{-1}$ from W78 on the Sun. Abundances early in the event indicate a temperature of $2.5 \mathrm{MK}$, but the proton excess is quite modest, perhaps a factor of two above expectations. By the middle of the event the temperature rises slightly to $3.2 \mathrm{MK}$ and the factor-of-ten proton excess is seen.

The final three periods shown in Figure 8e illustrate that temperature determination becomes impossible when the abundance enhancements are flat, independent of $A / Q$. We can measure temperatures when there is either increasing or decreasing dependence on $A / Q$, 

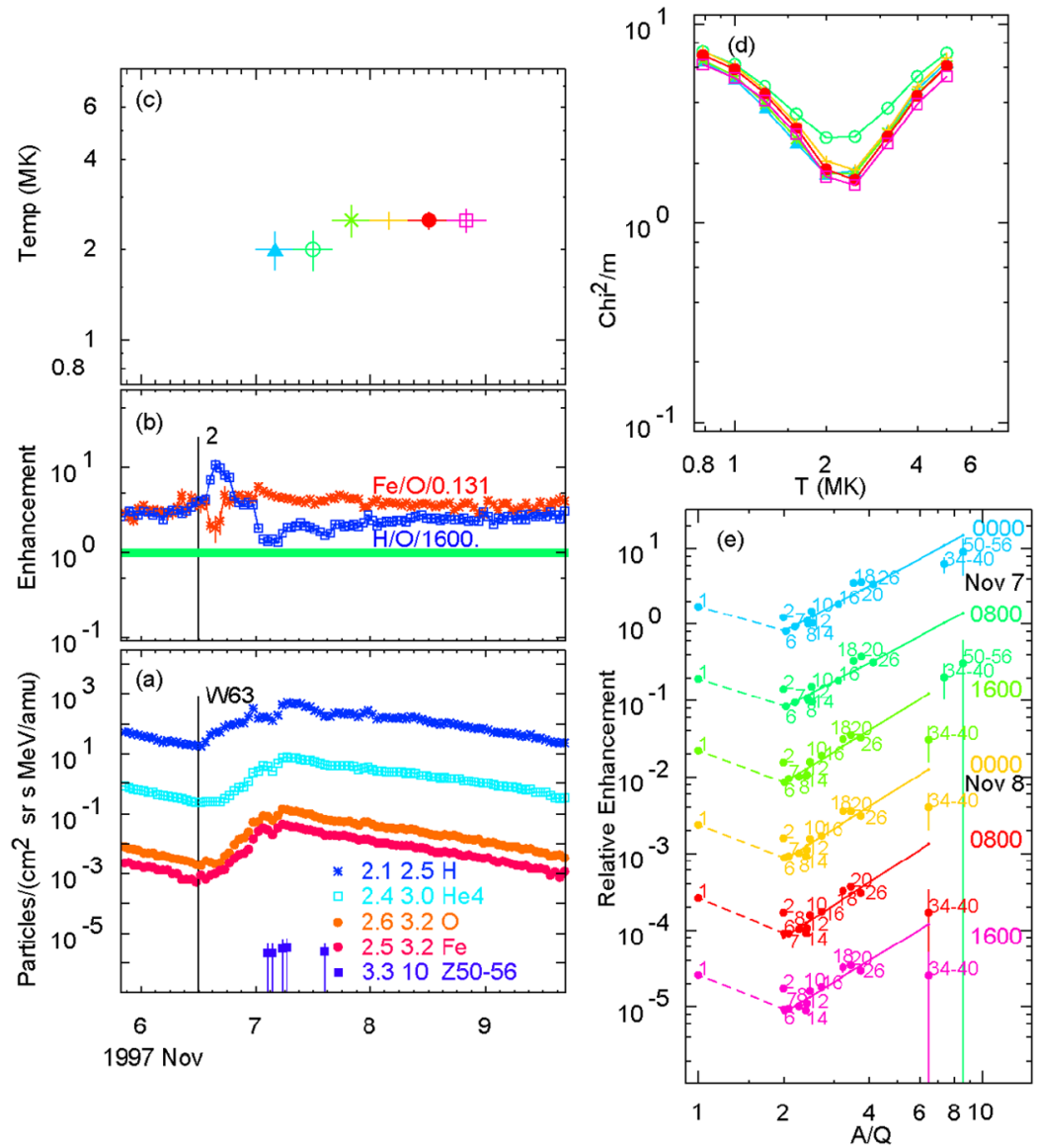

Figure 5 (a) Intensities of $\mathrm{H}, \mathrm{He}, \mathrm{O}, \mathrm{Fe}$, and $50 \leq Z \leq 56$ ions versus time. (b) Normalized abundance enhancements $\mathrm{H} / \mathrm{O}$ and $\mathrm{Fe} / \mathrm{O}$ versus time. (c) Temperatures versus time for the 6 November 1997 SEP event. (d) Shows $\chi^{2} / m$ versus $T$ for each 8 -hr interval. (e) Shows enhancements, labeled by $Z$ versus $A / Q$ for each 8-hr interval shifted $\times 0.1$ with best-fit power law for elements with $Z \geq 6$ (solid) joined to $\mathrm{H}$ by dashed lines. Colors correspond to the six intervals in (c), (d), and (e) and symbols in (c) and (d); times are also listed in (e). Dashed lines join $\mathrm{H}$ with its associated elements in (e). Event onset is flagged with solar longitude in (a) and event number from Reames (2016a) in (b).

but we cannot determine $A / Q$ or $T$ when there is no variation and the enhancements are independent of $A / Q$. Nevertheless, it is clear that the proton enhancements are a factor of ten above the other elements here as well.

Figure 9 shows the event of 29 September 2013 from W33 with a CME speed of $1179 \mathrm{~km} \mathrm{~s}^{-1}$. This event shows the classic evolution where $\mathrm{Fe} / \mathrm{O}$ decreases with time.

The event in Figure 9 shows a consistent temperature of $1.5 \mathrm{MK}$ indicating a lack of hotter material with impulsive suprathermal ions, yet the proton enhancement is significantly above expectation early, when the enhancements are rising with $A / Q$, but are well within expectations when the enhancements are falling with $A / Q$.

Does the large proton excess depend upon the presence of impulsive material or on the slope of the power law? Figure 9e suggests that only the slope matters, but Figure 8e shows predicted proton abundances for both positive slope and $T=2.5 \mathrm{MK}$. 

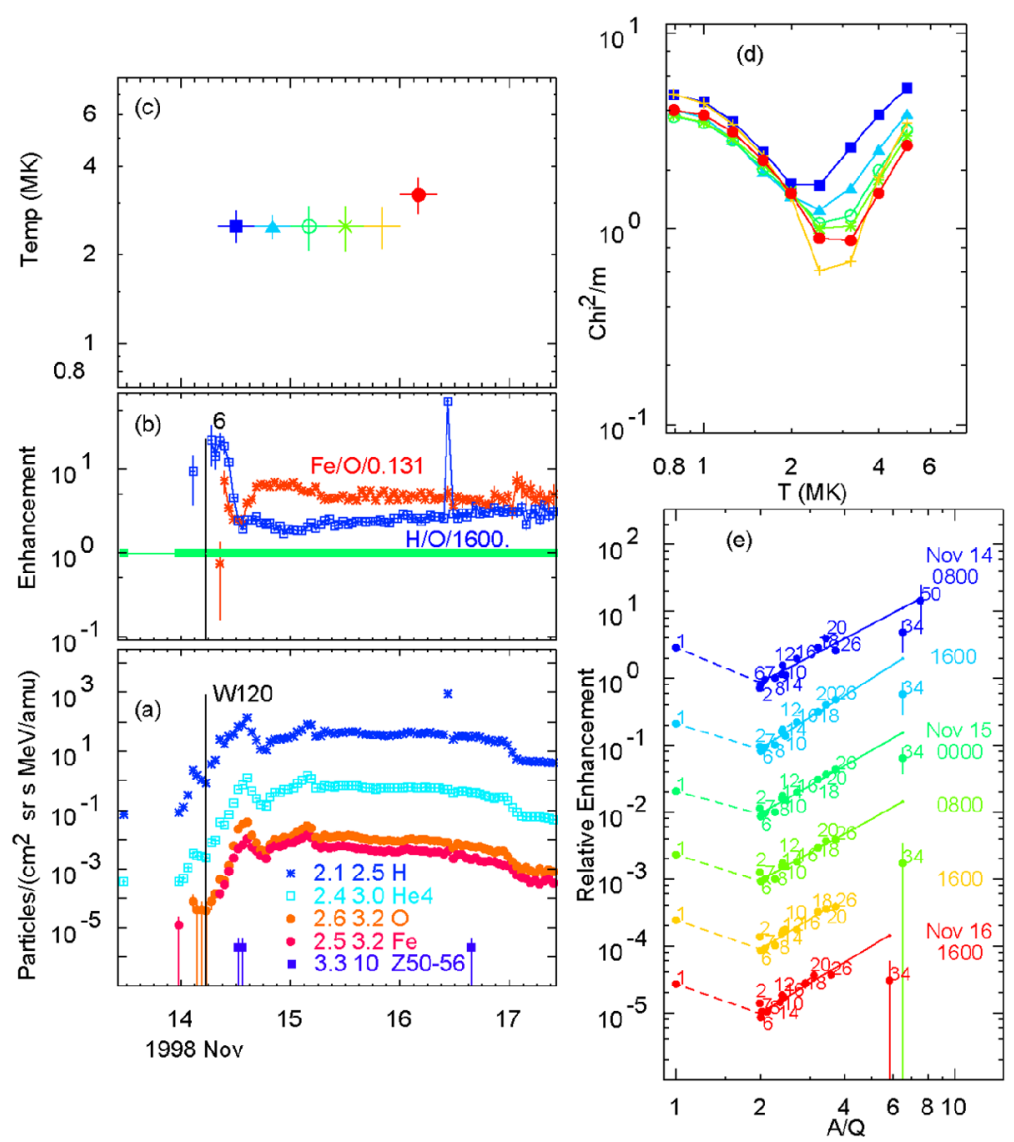

Figure 6 (a) Intensities of $\mathrm{H}, \mathrm{He}, \mathrm{O}, \mathrm{Fe}$, and $50 \leq Z \leq 56$ ions versus time. (b) Normalized abundance enhancements $\mathrm{H} / \mathrm{O}$ and $\mathrm{Fe} / \mathrm{O}$ versus time. (c) Temperatures versus time for the 14 November 1998 SEP event. (d) Shows $\chi^{2} / m$ versus $T$ for each 8 -hr interval. (e) Shows enhancements, labeled by $Z$ versus $A / Q$ for each 8-hr interval shifted $\times 0.1$ with best-fit power law for elements with $Z \geq 6$ (solid) joined to $\mathrm{H}$ by dashed lines. Colors correspond to the six intervals in (c), (d), and (e) and symbols in (c) and (d); times are also listed in (e). Dashed lines join $\mathrm{H}$ with its associated elements in (e). Event onset is flagged with solar longitude in (a) and event number from Reames (2016a) in (b).

\section{Proton Excess and Distributions in $A / Q$ and $T$}

We have seen several examples of events that show a typical behavior of proton enhancement in gradual SEP events and a few events that show an atypical behavior. What remains is to examine the probability distributions for a wide range of cases. Since the behavior can vary with time during an event we must look at the distributions of the basic 8-hr averages, nearly 400 intervals within the SEP events originally studied by Reames (2016a).

The upper panel of Figure 10 shows the distribution of proton enhancements during the 8 -hr intervals as a function of the slope, or power of $A / Q$, of the fit. Clearly, the peak of the distribution lies at a negative slope near -1 , where most intervals have a proton enhancement within a factor of about three of having the value correctly predicted by the ions with $Z \geq 6$ or modest excesses. Grouping the intervals by temperature, in the lower panels of Figure 10, shows that intervals with $T \geq 2.5$, on the right, identify with the posi- 


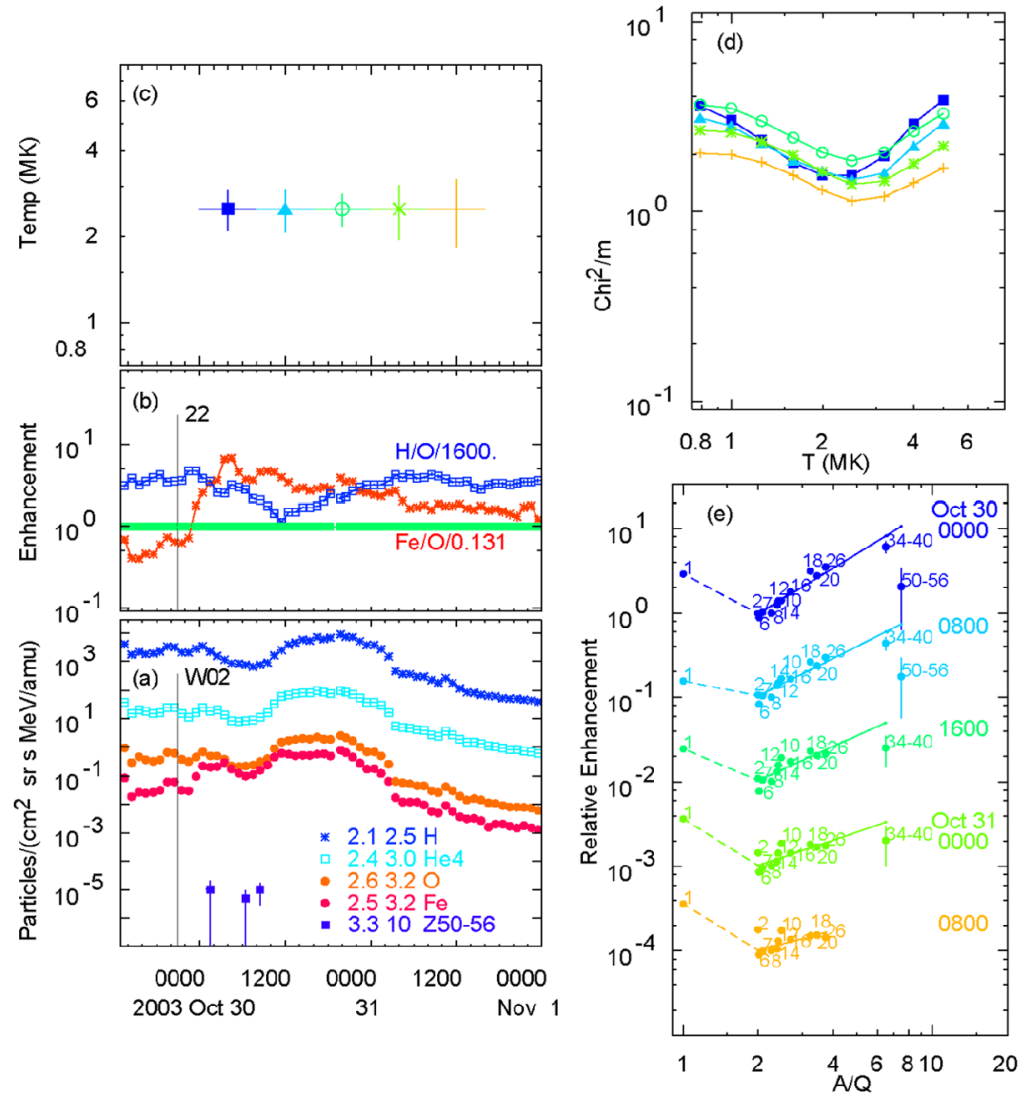

Figure 7 (a) Intensities of $\mathrm{H}, \mathrm{He}, \mathrm{O}, \mathrm{Fe}$, and $50 \leq Z \leq 56$ ions versus time. (b) Normalized abundance enhancements $\mathrm{H} / \mathrm{O}$ and $\mathrm{Fe} / \mathrm{O}$ versus time. (c) Temperatures are shown versus time for the 29 October 2003 SEP event. (d) Shows $\chi^{2} / m$ versus $T$ for each 8 -hr interval. (e) Shows enhancements, labeled by $Z$ versus $A / Q$ for each 8 -hr interval shifted $\times 0.1$ with best-fit power law for elements with $Z \geq 6$ (solid) joined to $\mathrm{H}$ by dashed lines. Colors correspond to the five intervals in (c), (d), and (e) and symbols in (c) and (d); times are also listed in (e). Dashed lines join $\mathrm{H}$ with its associated elements in (e). Event onset is flagged with solar longitude in (a) and event number from Reames (2016a) in (b).

tive slope involving reaccelerated impulsive ions with typical power-of-ten proton excesses. Lower temperatures of the ambient coronal plasma in the lower left panel have a much better chance of predicting the proton intensity using only the $Z \geq 6$ ions, but there are some event intervals with positive slope that usually occur early in these events. In the original temperature distribution of events (Reames, 2016a), which is unchanged here, $24 \%(11 / 45)$ of the events were in the 2.5-3.2 MK interval and $69 \%(31 / 45)$ had $T \leq 1.6 \mathrm{MK}$. The proton excesses tend to follow that division, not because of the temperature itself but, more likely, because of the associated distribution of positive and negative slope of $A / Q$.

\section{Discussion}

The abundances we study depend upon two underlying factors. First, elements suffer the FIP effect as they are brought into the corona to produce the reference SEP abundances 


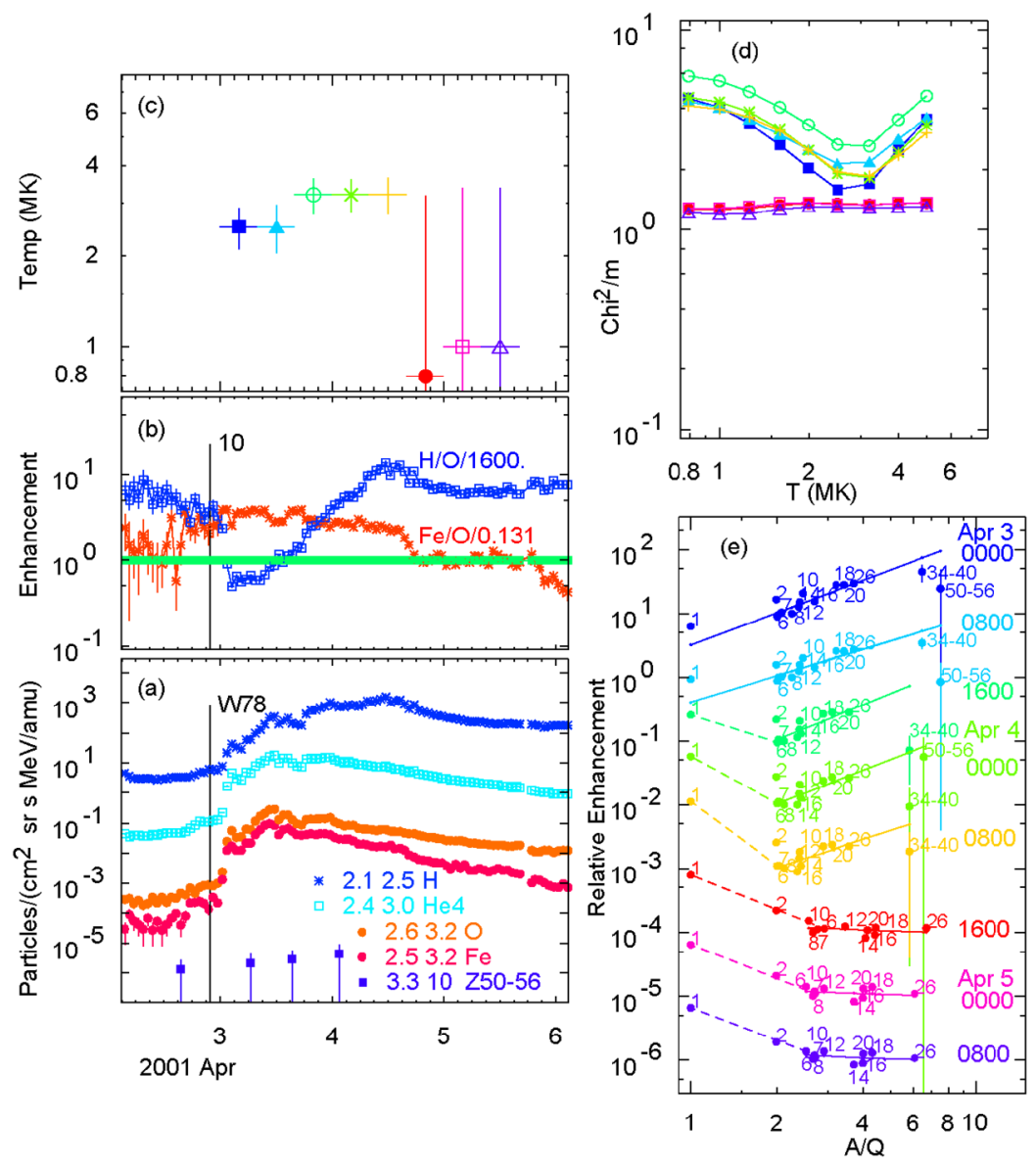

Figure 8 (a) Intensities of $\mathrm{H}, \mathrm{He}, \mathrm{O}, \mathrm{Fe}$, and $50 \leq Z \leq 56$ ions versus time. (b) Normalized abundance enhancements $\mathrm{H} / \mathrm{O}$ and $\mathrm{Fe} / \mathrm{O}$ versus time. (c) Temperatures are shown versus time for the 2 April 2001 SEP event. (d) Shows $\chi^{2} / m$ versus $T$ for each 8-hr interval. (e) Shows enhancements, labeled by $Z$ versus $A / Q$ for each 8-hr interval shifted $\times 0.1$ with best-fit power law for elements with $Z \geq 6$ extrapolated down to $\mathrm{H}$ at $A / Q=1$ (solid) or joined to $\mathrm{H}$ by dashed lines. Colors correspond to the eight intervals in (c), (d), and (e) and symbols in (c) and (d); times are also listed in (e). Dashed lines join $\mathrm{H}$ with its associated elements in (e). Event onset is flagged with solar longitude in (a) and event number from Reames (2016a) in (b).

tabulated in the Appendix. Second, their acceleration and transport depend, not only upon particle rigidity, but approximately as a power law on rigidity. Similar processes apply, not only to SEPs, but even to energetic ions in corotating interaction regions where shock waves accelerate ions, from the solar wind in this case, to produce enhancements that usually decline with $A / Q$ (Reames, 2018c) like most SEP events here. All ions experience this process, but the protons in shock acceleration have a dual role; they are not only accelerated like other ions, but they must generate waves as they stream away so as to scatter subsequent ions back and forth across the shock (Bell, 1978; Lee, 1983, 2005; Ng and Reames, 2008; Desai and Giacalone, 2016). Are extra protons sometimes required for the latter task? If we adjust the abundance normalization from $\mathrm{O}$ to $\mathrm{H}$, events with formerly "excess protons" are now seen as unable to accelerate the expected number of heavy ions given the available 

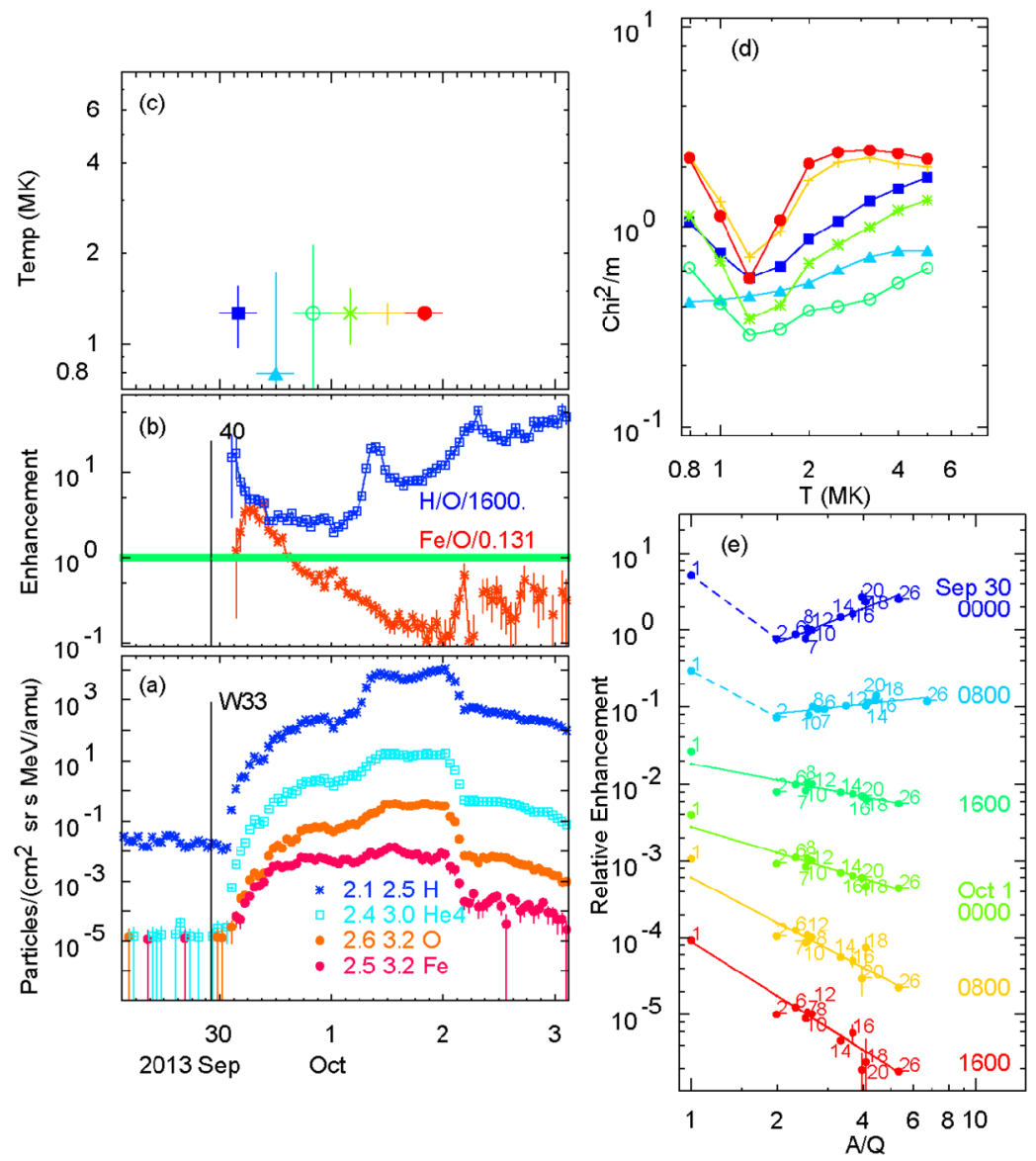

Figure 9 (a) Intensities of $\mathrm{H}, \mathrm{He}, \mathrm{O}$, and $\mathrm{Fe}$ ions versus time. (b) Normalized abundance enhancements $\mathrm{H} / \mathrm{O}$ and $\mathrm{Fe} / \mathrm{O}$ versus time. (c) Temperatures are shown versus time for the 29 September 2013 SEP event. (d) Shows $\chi^{2} / m$ versus $T$ for each 8 -hr interval. (e) Shows enhancements, labeled by $Z$ versus $A / Q$ for each 8-hr interval shifted $\times 0.1$ with best-fit power law for elements with $Z \geq 6$ extrapolated down to $\mathrm{H}$ at $A / Q=1$ (solid) or joined to $\mathrm{H}$ by dashed lines. Colors correspond to the six intervals in (c), (d), and (e) and symbols in (c) and (d); times are also listed in (e). Dashed lines join $\mathrm{H}$ with its associated elements in (e). Event onset is flagged with solar longitude in (a) and event number from Reames (2016a) in (b).

protons and the wave spectrum they have produced. However, we might not be surprised to find excess protons needed early in events when the shocks are strong and resonant waves are just forming, but less later as the shocks weaken and the pattern of waves is established (see also Reames, Ng, and Tylka, 2000).

When we compare ions at the same velocity, as we do for studies of abundance enhancements, the ions interact with different parts of the proton-generated wave spectrum. For example, for $2.5 \mathrm{MeV}$ protons the wave spectrum is approximately generated by streaming $2.5 \mathrm{MeV}$ protons, for $2.5 \mathrm{MeV} \mathrm{amu}^{-1} \mathrm{He}, \mathrm{C}$, or $\mathrm{O}$ with $A / Q=2$ the spectrum is generated by streaming $10 \mathrm{MeV}$ protons, for $2.5 \mathrm{MeV} \mathrm{amu}^{-1} \mathrm{Fe}$ at $A / Q=4$ the spectrum is generated by streaming $39 \mathrm{MeV}$ protons, and for $2.5 \mathrm{MeV} \mathrm{amu}^{-1}$ heavy ions with $A / Q=10$ the spectrum is generated by streaming $224 \mathrm{MeV}$ protons. Thus the proton spectrum and the slope of the $A / Q$-dependence of heavy ion enhancements are related. Can a proton spectrum, which 

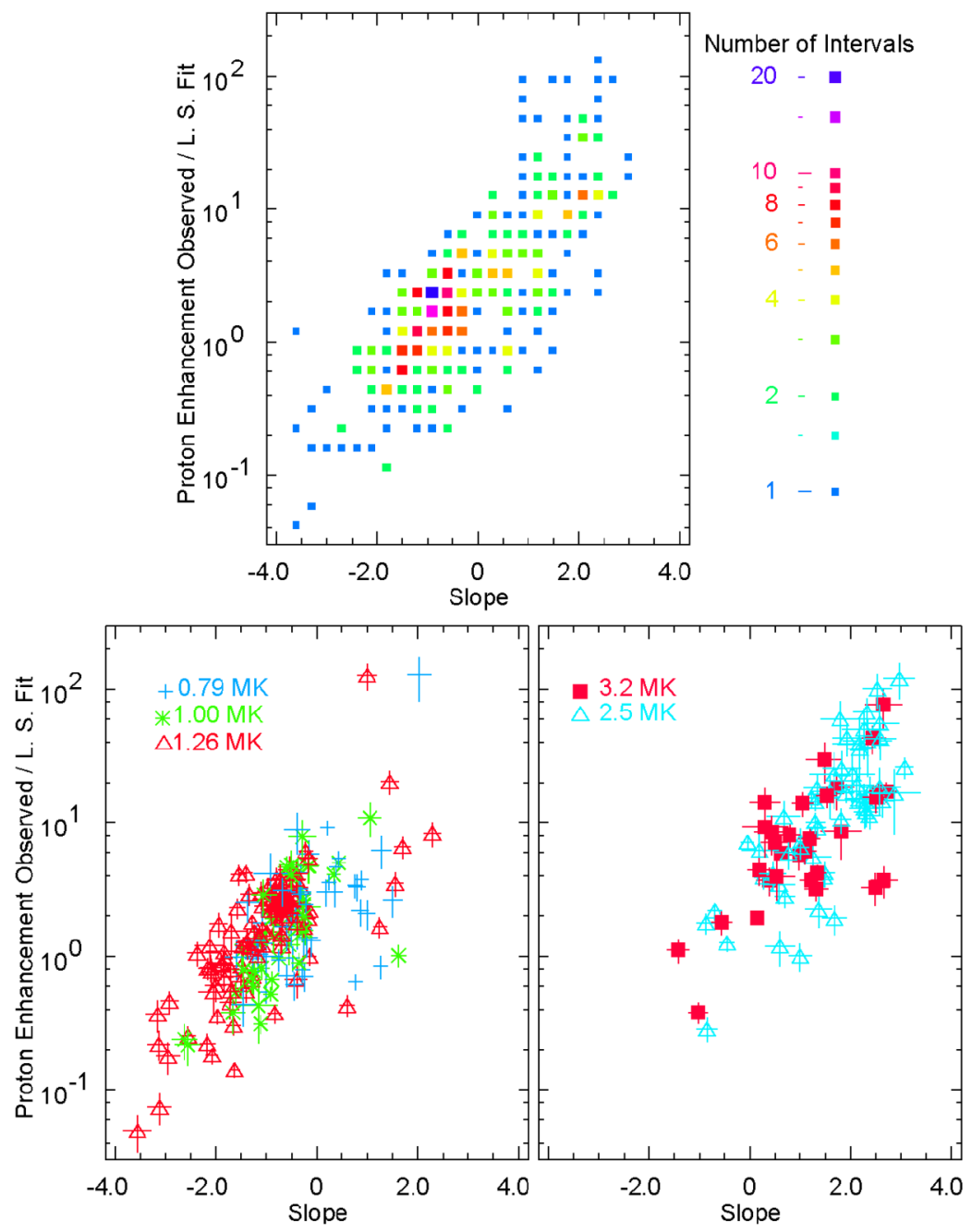

Figure 10 In all panels, the enhancement of $\mathrm{H}$ relative to that expected from the least-squares (L.S.) power-law fit of elements $Z \geq 6$ is shown versus the slope or power of $A / Q$ from the fit of elements $Z \geq 6$. The upper panel shows a histogram of the distribution of all 398 8-hr intervals in this space with symbol color and size showing the number at each location. The lower left panel shows the distribution of intervals with $T=0.79,1.0$, and $1.26 \mathrm{MK}$. The lower right panel shows the distribution of intervals with $T=2.5$ and $3.2 \mathrm{MK}$.

usually declines with energy, produce enough waves to accelerate heavier ion abundances that increase with resonant rigidity? For positive slopes in $A / Q$, perhaps we should compare $39 \mathrm{MeV}$ protons with $2.5 \mathrm{MeV} a m u^{-1} \mathrm{Fe}$. Unfortunately, $39 \mathrm{MeV}$ proton data are not available during these events.

The proton spectral shape can be important, and any downward breaks in the proton spectrum will especially tend to depress heavy ions perhaps such as may be occurring for the $50 \leq Z \leq 56$ ions in Figures 7 and 8. Breaks can also disrupt the power law lower in $A / Q$ as seen by the break at $\mathrm{Mg}$ in the multi-spacecraft study of the SEP event of 23 January 2012 (Reames, 2017b). 
Do the excess protons come from the same ion population as the heavy ions? Recently, Reames (2019b) suggested that two components contributed to the shock acceleration in impulsive SEP events with significant excess protons, the heavy ions may come from residual impulsive suprathermal ions with positive power of $A / Q$, while the protons come from the ambient plasma with a negative power of $A / Q$ (see Figure 9 in Reames, 2019b). However, excess protons are seen rather often and in similar amounts, making this dual-source explanation less probable, since two components might be expected to produce a larger range of relative variations. Also, two components would not explain the event in Figure 9 where a proton excess and positive power of $A / Q$ coexist for ions with an ambient $1.5 \mathrm{MK}$ source plasma temperature. Apparently, more protons are required to support shock conditions that can produce or can maintain a strong positive power of $A / Q$ in the enhancements of heavy elements, even when injected abundances already have a positive power of $A / Q$ initially. However, cases do exist where positive powers of $A / Q$ extrapolate to predict the observed proton enhancement (Figures 4 and 8).

We summarize the general behavior of gradual SEP events as follows:

i) Most time intervals in most gradual SEP events ( $>60 \%$ ) have source temperatures $0.7 \leq$ $T \leq 1.6 \mathrm{MK}$ and abundances that decrease as a power of $A / Q$. Proton enhancements are predicted by the power-law pattern of the other elements.

ii) Time periods during those events $(>20 \%)$ dominated by pre-accelerated impulsive ions, with $T>2 \mathrm{MK}$ and abundances that increase as a power of $A / Q$, usually have proton enhancements a factor of about ten above the expected values.

When we consider impulsive and gradual SEP events together, three situations can exist. Proton abundances can be consistent with the expectations of a power law in $A / Q$ in i) "pure" small Fe-rich impulsive SEP events (Reames, 2019b) and in ii) "pure" large gradual SEP events with decreasing powers of $A / Q$ with few impulsive ions. However, proton abundances significantly exceed expectations primarily in iii) "compound" SEP events where shock waves reaccelerate impulsive SEP material. This new three-way behavior of proton abundances can help identify the physical processes involved. Thus, Fe-rich SEPs with expected proton abundances are probably accelerated in a magnetic reconnection region without involvement of shocks, while Fe-rich SEPs with a 10-fold excess of protons are probably reaccelerated by a shock wave. This is a powerful new insight for impulsive SEP events.

Is there a difference between the impulsive events where proton intensities are boosted by the narrow shock wave from their associated CME and the gradual events where fast, wide shock waves predominantly sample impulsive suprathermal seed populations? Perhaps there is, since the shock in the former samples a single impulsive injection while the latter can average over the cumulative residual ions from many impulsive events, greatly averaging and reducing the event-to-event abundance variations as discussed by Reames (2016b).

In contrast to $\mathrm{H}$, He shows substantial source abundance variations $30 \leq \mathrm{He} / \mathrm{O} \leq 100$ in both impulsive and gradual events (Reames, 2017c, 2019a) with a few impulsive SEP events showing extreme suppression of $\mathrm{He} / \mathrm{O} \approx 2$ (Reames, 2019a). These variations in $\mathrm{He}$ may result from FIP-related inefficient ionization of He during transport up into the corona (Laming, 2009). $\mathrm{H}$ and $\mathrm{He}$ do not share the same behavior; this is why we study $\mathrm{H} / \mathrm{O}$ and $\mathrm{He} / \mathrm{O}$ rather than $\mathrm{He} / \mathrm{H}$. In view of the comparisons with other elements (e.g. Reames, 2018a), it is not surprising that variations of $\mathrm{H}$ and He in SEP events also seem to differ completely from variations of $\mathrm{H}$ and $\mathrm{He}$ in the solar wind.

Further progress in understanding proton abundances may depend upon theory and modeling that includes other parameters that can affect acceleration and transport. Do the current 
models of SEP acceleration and transport support the observed $A / Q$-dependence? Do they predict excess protons? The goal of this article has been to examine SEP events from a new perspective and to show the range of variations and suggest the probable cause. More detailed theoretical studies relating proton spectra to $A / Q$-dependence are needed.

Disclosure of Potential Conflicts of Interest The author declares to have no conflicts of interest.

Publisher's Note Springer Nature remains neutral with regard to jurisdictional claims in published maps and institutional affiliations.

Open Access This article is distributed under the terms of the Creative Commons Attribution 4.0 International License (http://creativecommons.org/licenses/by/4.0/), which permits unrestricted use, distribution, and reproduction in any medium, provided you give appropriate credit to the original author(s) and the source, provide a link to the Creative Commons license, and indicate if changes were made.

\section{Appendix: Reference Abundances of Elements}

The average element abundances in gradual SEP events are a measure of the coronal abundances sampled by SEP events (reference gradual SEPs in Table 1). They differ from photospheric abundances (Table 1) by a factor which depends upon FIP (e.g. Reames, 2018a, 2018b). Ion "enhancements" are defined as the observed abundance of a species, relative to $\mathrm{O}$, divided by the reference abundance of that species, relative to $\mathrm{O}$.

Table 1 Reference gradual SEP abundances, photospheric, and impulsive SEP abundances are shown for various elements.

\begin{tabular}{llllll}
\hline & $Z$ & FIP $[\mathrm{eV}]$ & Photosphere $^{1}$ SEPs $^{2}$ & Reference gradual SEPs & Avg. impulsive \\
\hline $\mathrm{H}$ & 1 & 13.6 & $(1.74 \pm 0.17) \times 10^{6 *}$ & $(1.6 \pm 0.2) \times 10^{6}$ & - \\
$\mathrm{He}$ & 2 & 24.6 & $156000 \pm 7000$ & $57000 \pm 5000$ & $53000 \pm 3000^{* *}$ \\
$\mathrm{C}$ & 6 & 11.3 & $550 \pm 76^{*}$ & $420 \pm 10$ & $386 \pm 8$ \\
$\mathrm{~N}$ & 7 & 14.5 & $126 \pm 35^{*}$ & $128 \pm 8$ & $139 \pm 4$ \\
$\mathrm{O}$ & 8 & 13.6 & $1000 \pm 161^{*}$ & $1000 \pm 10$ & $1000 \pm 10$ \\
$\mathrm{Ne}$ & 10 & 21.6 & $210 \pm 54$ & $157 \pm 10$ & $478 \pm 24$ \\
$\mathrm{Mg}$ & 12 & 7.6 & $64.5 \pm 9.5$ & $178 \pm 4$ & $404 \pm 30$ \\
$\mathrm{Si}$ & 14 & 8.2 & $61.6 \pm 9.1$ & $151 \pm 4$ & $325 \pm 12$ \\
$\mathrm{~S}$ & 16 & 10.4 & $25.1 \pm 2.9^{*}$ & $25 \pm 2$ & $84 \pm 4$ \\
$\mathrm{Ar}$ & 18 & 15.8 & $5.9 \pm 1.5$ & $4.3 \pm 0.4$ & $34 \pm 2$ \\
$\mathrm{Ca}$ & 20 & 6.1 & $4.0 \pm 0.7$ & $11 \pm 1$ & $85 \pm 4$ \\
$\mathrm{Fe}$ & 26 & 7.9 & $57.6 \pm 8.0^{*}$ & $131 \pm 6$ & $1170 \pm 48$ \\
$\mathrm{Zn}-\mathrm{Zr}$ & $30-40$ & - & - & $0.04 \pm 0.01$ & $2.0 \pm 0.2$ \\
$\mathrm{Sn}-\mathrm{Ba}$ & $50-56$ & - & - & $0.0066 \pm 0.001$ & $2.0 \pm 2$ \\
$\mathrm{Os}-\mathrm{Pb}$ & $76-82$ & - & - & $0.0007 \pm 0.0003$ & $0.64 \pm 0.12$ \\
\hline
\end{tabular}

\footnotetext{
${ }^{1}$ Lodders, Palme, and Gail (2009); see also Asplund et al. (2009)

${ }^{2}$ Reames (1995, 2014, 2017a)

${ }^{3}$ Reames, Cliver, and Kahler (2014a)

* Caffau et al. (2011)

*** Reames (2019a)
} 


\section{References}

Asplund, M., Grevesse, N., Sauval, A.J., Scott, P.: 2009, The chemical composition of the Sun. Annu. Rev. Astron. Astrophys. 47, 481. DOI.

Bell, A.R.: 1978, The acceleration of cosmic rays in shock fronts. I. Mon. Not. Roy. Astron. Soc. $182,147$. DOI.

Breneman, H.H., Stone, E.C.: 1985, Solar coronal and photospheric abundances from solar energetic particle measurements. Astrophys. J. Lett. 299, L57. DOI.

Bučík, R., Innes, D.E., Mall, U., Korth, A., Mason, G.M., Gómez-Herrero, R.: 2014, Multi-spacecraft observations of recurrent ${ }^{3} \mathrm{He}$-rich solar energetic particles. Astrophys. J. 786, 71. DOI.

Bučík, R., Innes, D.E., Chen, N.H., Mason, G.M., Gómez-Herrero, R., Wiedenbeck, M.E.: 2015, Long-lived energetic particle source regions on the Sun. J. Phys. Conf. Ser. 642, 012002. DOI.

Bučík, R., Innes, D.E., Mason, G.M., Wiedenbeck, M.E., Gómez-Herrero, R., Nitta, N.V.: 2018, ${ }^{3}$ He-rich solar energetic particles in helical jets on the Sun. Astrophys. J. 852, 76. DOI.

Caffau, E., Ludwig, H.-G., Steffen, M., Freytag, B., Bonofacio, P.: 2011, Solar chemical abundances determined with a CO5BOLD 3D model atmosphere. Solar Phys. 268, 255. DOI.

Chen, N.H., Bučík, R., Innes, D.E., Mason, G.M.: 2015, Case studies of multi-day ${ }^{3}$ He-rich solar energetic particle periods. Astron. Astrophys. 580, 16. DOI.

Cliver, E.W., Kahler, S.W., Reames, D.V.: 2004, Coronal shocks and solar energetic proton events. Astrophys. J. 605, 902. DOI.

Cohen, C.M.S., Mason, G.M., Mewaldt, R.A.: 2017, Characteristics of solar energetic ions as a function of longitude. Astrophys. J. 843, 132. DOI.

Cohen, C.M.S., Stone, E.C., Mewaldt, R.A., Leske, R.A., Cummings, A.C., Mason, G.M., Desai, M.I., von Rosenvinge, T.T., Wiedenbeck, M.E.: 2005, Heavy ion abundances and spectra from the large solar energetic particle events of October-November 2003. J. Geophys. Res. 110, A916. DOI.

Desai, M.I., Giacalone, J.: 2016, Large gradual solar energetic particle events. Living Rev. Solar Phys. DOI.

Desai, M.I., Mason, G.M., Dwyer, J.R., Mazur, J.E., Gold, R.E., Krimigis, S.M., Smith, C.W., Skoug, R.M.: 2003, Evidence for a suprathermal seed population of heavy ions accelerated by interplanetary shocks near 1 AU. Astrophys. J. 588, 1149. DOI.

Drake, J.F., Cassak, P.A., Shay, M.A., Swisdak, M., Quataert, E.: 2009, A magnetic reconnection mechanism for ion acceleration and abundance enhancements in impulsive flares. Astrophys. J. Lett. 700, L16. DOI.

Gopalswamy, N., Xie, H., Yashiro, S., Akiyama, S., Mäkelä, P., Usoskin, I.G.: 2012, Properties of ground level enhancement events and the associated solar eruptions during Solar Cycle 23. Space Sci. Rev. 171, 23. DOI.

Gosling, J.T.: 1993, The solar flare myth. J. Geophys. Res. 98, 18937. DOI.

Kahler, S.W., Reames, D.V., Sheeley, N.R. Jr.: 2001, Coronal mass ejections associated with impulsive solar energetic particle events. Astrophys. J. 562, 558. DOI.

Kahler, S.W., Sheeley, N.R. Jr., Howard, R.A., Koomen, M.J., Michels, D.J., McGuire, R.E., von Rosenvinge, T.T., Reames, D.V.: 1984, Associations between coronal mass ejections and solar energetic proton events. J. Geophys. Res. 89, 9683. DOI.

Kasper, J.C., Stevens, M.L., Lazarus, A.J., Steinberg, J.T., Ogilvie, K.W.: 2007, Solar wind helium abundance as a function of speed and heliographic latitude: variation through a solar cycle. Astrophys. J. 660, 901. DOI.

Laming, J.M.: 2009, Non-WKB models of the first ionization potential effect: implications for solar coronal heating and the coronal helium and neon abundances. Astrophys. J. 695, 954. DOI.

Laming, J.M.: 2015, The FIP and inverse FIP effects in solar and stellar coronae. Living Rev. Solar Phys. 12, 2. DOI.

Lee, M.A.: 1983, Coupled hydromagnetic wave excitation and ion acceleration at interplanetary traveling shocks. J. Geophys. Res. 88, 6109. DOI.

Lee, M.A.: 2005, Coupled hydromagnetic wave excitation and ion acceleration at an evolving coronal/interplanetary shock. Astrophys. J. Suppl. 158, 38. DOI.

Lee, M.A., Mewaldt, R.A., Giacalone, J.: 2012, Shock acceleration of ions in the heliosphere. Space Sci. Rev. 173, 247. DOI.

Lodders, K., Palme, H., Gail, H.-P.: 2009, Abundances of the elements in the solar system. In: Trümper, J.E. (ed.) Landolt-Börnstein, New Series, Springer, Berlin, 560.

Luhn, A., Klecker, B., Hovestadt, D., Gloeckler, G., Ipavich, F.M., Scholer, M., Fan, C.Y., Fisk, L.A.: 1984, Ionic charge states of $\mathrm{N}, \mathrm{Ne}, \mathrm{Mg}, \mathrm{Si}$ and $\mathrm{S}$ in solar energetic particle events. Adv. Space Res. 4, 161. DOI.

Mason, G.M.: 2007, ${ }^{3}$ He-rich solar energetic particle events. Space Sci. Rev. 130, 231. DOI.

Mason, G.M., Mazur, J.E., Dwyer, J.R.: 2002, A new heavy ion abundance enrichment pattern in ${ }^{3} \mathrm{He}$-rich solar particle events. Astrophys. J. Lett. 565, L51. DOI. 
Mason, G.M., Ng, C.K., Klecker, B., Green, G.: 1989, Impulsive acceleration and scatter-free transport of about $1 \mathrm{MeV}$ per nucleon ions in ${ }^{3} \mathrm{He}$-rich solar particle events. Astrophys. J. 339, 529. DOI.

Mason, G.M., Mazur, J.E., Dwyer, J.R., Jokippi, J.R., Gold, R.E., Krimigis, S.M.: 2004, Abundances of heavy and ultraheavy ions in ${ }^{3} \mathrm{He}$-rich solar flares. Astrophys. J. 606, 555. DOI.

Mazzotta, P., Mazzitelli, G., Colafrancesco, S., Vittorio, N.: 1998, Ionization balance for optically thin plasmas: rate coefficients for all atoms and ions of the elements H to Ni. Astron. Astrophys. Suppl. Ser. 133, 403. DOI.

Mewaldt, R.A., Cohen, C.M.S., Leske, R.A., Christian, E.R., Cummings, A.C., Stone, E.C., von Rosenvinge, T.T., Wiedenbeck, M.E.: 2002, Fractionation of solar energetic particles and solar wind according to first ionization potential. Adv. Space Res. 30, 79. DOI.

Mewaldt, R.A., Cohen, C.M.S., Labrador, A.W., Leske, R.A., Mason, G.M., Desai, M.I., Looper, M.D., Mazur, J.E., Selesnick, R.S., Haggerty, D.K.: 2005, Proton, helium, and electron spectra during the large solar particle events of October-November 2003. J. Geophys. Res. 110, A09S18. DOI.

Meyer, J.-P.: 1985, The baseline composition of solar energetic particles. Astrophys. J. Suppl. 57, 151. DOI.

Ng, C.K., Reames, D.V.: 2008, Shock acceleration of solar energetic protons: the first 10 minutes. Astrophys. J. Lett. 686, L123. DOI.

Ng, C.K., Reames, D.V., Tylka, A.J.: 1999, Effect of proton-amplified waves on the evolution of solar energetic particle composition in gradual events. Geophys. Res. Lett. 26, 2145. DOI.

Ng, C.K., Reames, D.V., Tylka, A.J.: 2001, Abundances, spectra, and anisotropies in the 1998 Sep 30 and 2000 Apr 4 large SEP events. Proc. 27th Int. Cosmic-Ray Conf., 8, 3140.

Ng, C.K., Reames, D.V., Tylka, A.J.: 2003, Modeling shock-accelerated solar energetic particles coupled to interplanetary Alfvén waves. Astrophys. J. 591, 461. DOI.

Ng, C.K., Reames, D.V., Tylka, A.J.: 2012, Solar energetic particles: shock acceleration and transport through self-amplified waves. AIP Conf. Proc. 1436, 212. DOI.

Parker, E.N.: 1963, Interplanetary Dynamical Processes, Interscience, New York.

Reames, D.V.: 1988, Bimodal abundances in the energetic particles of solar and interplanetary origin. Astrophys. J. Lett. 330, L71. DOI.

Reames, D.V.: 1995, Coronal abundances determined from energetic particles. Adv. Space Res. 15(7), 41.

Reames, D.V.: 1999, Particle acceleration at the Sun and in the heliosphere. Space Sci. Rev. 90, 413. DOI.

Reames, D.V.: 2000, Abundances of trans-iron elements in solar energetic particle events. Astrophys. J. Lett. 540, L111. DOI.

Reames, D.V.: 2009, Solar release times of energetic particles in ground-level events. Astrophys. J. 693, 812. DOI.

Reames, D.V.: 2013, The two sources of solar energetic particles. Space Sci. Rev. 175, 53. DOI.

Reames, D.V.: 2014, Element abundances in solar energetic particles and the solar corona. Solar Phys. 289, 977. DOI.

Reames, D.V.: 2015, What are the sources of solar energetic particles? Element abundances and source plasma temperatures. Space Sci. Rev. 194, 303. DOI.

Reames, D.V.: 2016a, Temperature of the source plasma in gradual solar energetic particle events. Solar Phys. 291, 911. DOI.

Reames, D.V.: 2016b, The origin of element abundance variations in solar energetic particles. Solar Phys. 291, 2099. DOI.

Reames, D.V.: 2017a, Solar Energetic Particles, Lect. Notes Phys. 932, Springer, Berlin. DOI. 978-3-31950870-2.

Reames, D.V.: 2017b, Spatial distribution of element abundances and ionization states in solar energeticparticle events. Solar Phys. 292, 133. DOI. arXiv.

Reames, D.V.: 2017c, The abundance of helium in the source plasma of solar energetic particles. Solar Phys. 292, 156. DOI. arXiv.

Reames, D.V.: 2018a, The "FIP effect" and the origins of solar energetic particles and of the solar wind. Solar Phys. 293, 47. DOI. arXiv.

Reames, D.V.: 2018b, Abundances, ionization states, temperatures, and FIP in solar energetic particles. Space Sci. Rev. 214, 61. DOI. arXiv.

Reames, D.V.: 2018c, Corotating shock waves and the solar-wind source of energetic ion abundances: power laws in $A / Q$. Solar Phys. 293, 144. DOI. arXiv.

Reames, D.V.: 2019a, Helium suppression in impulsive solar energetic-particle events. Solar Phys. $294,32$. DOI. arXiv.

Reames, D.V.: 2019b, Hydrogen and the abundances of elements in impulsive solar energetic-particle events. Solar Phys. 294, 37. DOI. arXiv.

Reames, D.V., Barbier, L.M., Ng, C.K.: 1996, The spatial distribution of particles accelerated by coronal mass ejection-driven shocks. Astrophys. J. 466, 473. DOI. 
Reames, D.V., Cliver, E.W., Kahler, S.W.: 2014a, Abundance enhancements in impulsive solar energeticparticle events with associated coronal mass ejections. Solar Phys. 289, 3817. DOI.

Reames, D.V., Cliver, E.W., Kahler, S.W.: 2014b, Variations in abundance enhancements in impulsive solar energetic-particle events and related CMEs and flares. Solar Phys. 289, 4675. DOI.

Reames, D.V., Cliver, E.W., Kahler, S.W.: 2015, Temperature of the source plasma for impulsive solar energetic particles. Solar Phys. 290, 1761. DOI.

Reames, D.V., Kahler, S.W., Ng, C.K.: 1997, Spatial and temporal invariance in the spectra of energetic particles in gradual solar events. Astrophys. J. 491, 414. DOI.

Reames, D.V., Meyer, J.P., von Rosenvinge, T.T.: 1994, Energetic-particle abundances in impulsive solar flare events. Astrophys. J. Suppl. 90, 649. DOI.

Reames, D.V., Ng, C.K.: 2004, Heavy-element abundances in solar energetic particle events. Astrophys. J. 610, 510. DOI.

Reames, D.V., Ng, C.K.: 2010, Streaming-limited intensities of solar energetic particles on the intensity plateau. Astrophys. J. 723, 1286. DOI.

Reames, D.V., Ng, C.K., Tylka, A.J.: 2000, Initial time dependence of abundances in solar particle events. Astrophys. J. Lett. 531, L83. DOI.

Reames, D.V., Stone, R.G.: 1986, The identification of solar ${ }^{3} \mathrm{He}$-rich events and the study of particle acceleration at the Sun. Astrophys. J. 308, 902. DOI.

Reames, D.V., von Rosenvinge, T.T., Lin, R.P.: 1985, Solar ${ }^{3}$ He-rich events and nonrelativistic electron events - a new association. Astrophys. J. 292, 716. DOI.

Reames, D.V., Barbier, L.M., von Rosenvinge, T.T., Mason, G.M., Mazur, J.E., Dwyer, J.R.: 1997, Energy spectra of ions accelerated in impulsive and gradual solar events. Astrophys. J. 483, 515. DOI.

Roth, I., Temerin, M.: 1997, Enrichment of ${ }^{3}$ He and heavy ions in impulsive solar flares. Astrophys. J. 477, 940. DOI.

Rouillard, A., Sheeley, N.R. Jr., Tylka, A., Vourlidas, A., Ng, C.K., Rakowski, C., Cohen, C.M.S., Mewaldt, R.A., Mason, G.M., Reames, D., et al.: 2012, The longitudinal properties of a solar energetic particle event investigated using modern solar imaging. Astrophys. J. 752, 44. DOI.

Schmelz, J.T., Reames, D.V., von Steiger, R., Basu, S.: 2012, Composition of the solar corona, solar wind, and solar energetic particles. Astrophys. J. 755, 33. DOI.

Serlemitsos, A.T., Balasubrahmanyan, V.K.: 1975, Solar particle events with anomalously large relative abundance of ${ }^{3}$ He. Astrophys. J. 198, 195. DOI.

Temerin, M., Roth, I.: 1992 , The production of ${ }^{3} \mathrm{He}$ and heavy ion enrichment in ${ }^{3} \mathrm{He}$-rich flares by electromagnetic hydrogen cyclotron waves. Astrophys. J. Lett. 391, L105. DOI.

Tylka, A.J., Lee, M.A.: 2006, Spectral and compositional characteristics of gradual and impulsive solar energetic particle events. Astrophys. J. 646, 1319. DOI.

Tylka, A.J., Cohen, C.M.S., Dietrich, W.F., Lee, M.A., Maclennan, C.G., Mewaldt, R.A., Ng, C.K., Reames, D.V.: 2005, Shock geometry, seed populations, and the origin of variable elemental composition at high energies in large gradual solar particle events. Astrophys. J. 625, 474. DOI.

von Rosenvinge, T.T., Barbier, L.M., Karsch, J., Liberman, R., Madden, M.P., Nolan, T., Reames, D.V., Ryan, L., Singh, S., Trexel, H.: 1995, The Energetic Particles: Acceleration, Composition, and Transport (EPACT) investigation on the Wind spacecraft. Space Sci. Rev. 71, 152. DOI.

Webber, W.R.: 1975, Solar and galactic cosmic ray abundances - a comparison and some comments. Proc. 14th Int. Cosmic Ray Conf., 5, 1597.

Zank, G.P., Rice, W.K.M., Wu, C.C.: 2000, Particle acceleration and coronal mass ejection driven shocks: a theoretical model. J. Geophys. Res. 105, 25079. DOI. 